\title{
A Novel Multiple-Output DUSTF Coding on High Mobility MIMO-Wireless Communication Systems
}

\author{
Rina Pudji Astuti ${ }^{1,2}$, Andriyan B. Suksmono ${ }^{1}$, Sugihartono ${ }^{1}$ \& Adit Kurniawan ${ }^{1}$ \\ ${ }^{1}$ School of the Electrical Engineering and Informatics - ITB - Indonesia \\ ${ }^{2}$ Department of Electrical Engineering - IT Telkom - Indonesia.
}

\begin{abstract}
In the future, wireless access system will operate in high data rate transmission and high mobility environment, to support private and public access. For such an environment, it is necessary to develop a system that has a higher spectrum efficiency and is able to mitigate selective fading problems. A novel multiple-output differential unitary space-time frequency (DUSTF) coding scheme is proposed to overcome those problems. The implementation of this inner coding scheme is unified with MIMO system, so that the scheme has a good spectrum efficiency. The differential space-time modulation in this proposed scheme is intended to operate in a non-coherent channel transmission scheme and to guarantee the system performance. In order to combat the selective fading problems, the multi-carrier space frequency scheme is utilized in the proposed scheme. In general, simulation result shows that the MIMO wireless system with the multiple-output DUSTF coding scheme in a noncoherent channel transmission scheme provides a good system performance. The proposed scheme can outperforms other previously published inner coding scheme for high mobility and high SNR. The system also achieves a good channel capacity.
\end{abstract}

Keywords: high data rate; multiple-output DUSTF coding; MIMO; wireless.

\section{Introduction}

The main problem in transmitting high data rate at high-speed mobility in wireless broadband access system is how to provide wide bandwidth in a rapidly changing channel condition [1]. However, the transmission of wide band information signal is restricted by limited bandwidth of the radio channel. A wide band transmitted signal is also disturbed by selective fading, resulting in difficulties in recovering these signals, so that cause the system performance degradation. Furthermore, it is not easy to know channel state information with a channel estimation scheme under this channel condition.

Generally, wireless communication researches offer partial solution to solve those problems. For examples, the solutions are to utilize a multiple antenna techniques due to its spectrum efficiency and a simple multi-carrier transmission scheme capable to overcome the selective fading condition. The 
implementation of non-coherent channel transmission scheme with differential modulation is also a good solution to overcome difficulties in estimating the response of the channel condition. These schemes could be applied regardless of the knowledge of the channel state [2]. Moreover, to improve the system performance degraded by the fading channel, the wireless broadband access system could utilize combination of an outer coding as channel coding and an inner coding, as illustrated in Figure 1, to enhance the performance of multiple transmit antennas system.

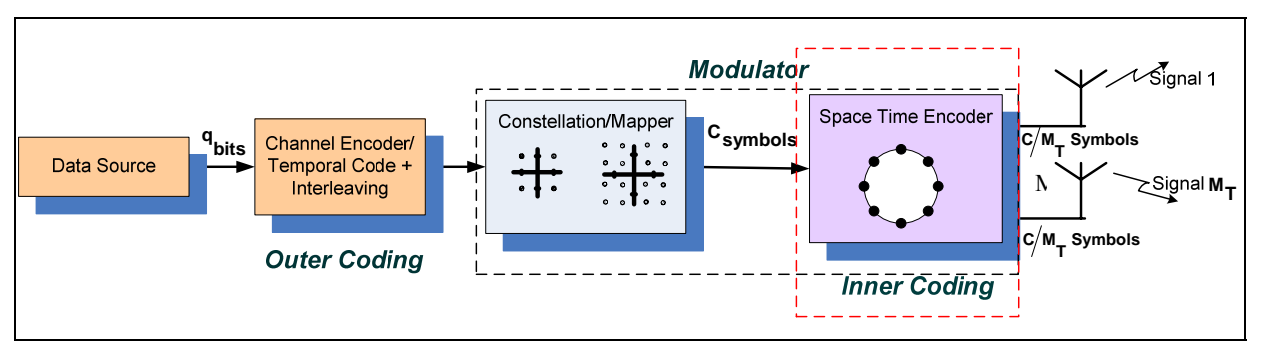

Figure 1 Outer and inner coding in MIMO-wireless communication system

Therefore, the MIMO system with such partial solutions can result in a nonoptimal performance. To get a comprehensive solution for high data rate - high mobility problems, we propose a multi-carrier differential inner coding scheme. In our previous research [2], we investigate the performance of three differential space-time schemes in wireless MIMO channel under similar conditions of mobile velocity, coding rate, frame size, modulation system, non-coherent transmission scheme and channel condition. It is shown that DUSTF coding scheme outperforms other differential space-time coding schemes, i.e. differential space-time block code (D-STBC) and differential unitary (Dunitary) coding. Furthermore, DUSTF coding performance with non-coherent transmission scheme is better performance than STBC with the perfect channel state information in low Eb/No, and vice versa.

However, in transmitting high data rate, some problems are encountered, such as the requirements of greater spectrum efficiency, higher processing gain and a faster signal processing as well. Those requirements are satisfied by applying parallel processing scheme. Hence, the proposed multiple output DUSTF coding scheme is extended from our previous research [2], i.e. the differential unitary space-time frequency (DUSTF) coding scheme. Except, the proposed scheme has the parallel/multiple output data, as initial inner code due to good processing gain. This scheme is to support parallel signal processing in the next step and non-coherent channel transmission scheme. 
Thus, we proposed generic model, where MIMO system with this scheme can be applied with an arbitrary $N_{R} \times M_{T}$ antennas system. i.e.: (i) $M_{T x}=N_{R x}=n_{s}$, (ii) $M_{T x}<N_{R x}$ with $n_{s}=N_{R x}$, and (iii) $M_{T x}>N_{R x} \cdot n_{s}$ is the number of the symbol. Then, each arm of the element transmit antenna employs space frequency scheme, which is based on OFDM technique as second code to support selective fading resiliency, to support high mobility and more spectrum efficiency. In this paper, we describe the analysis of the system performance, and channel capacity from multiple-output DUSTF coding in MIMO-wireless systems.

The rest of the paper is organized as follows. In Section 2, we investigate multiple-output DUSTF coding in MIMO-wireless systems with coherent channel or non-coherent channel transmission scheme. Next in Section 3, we discuss the system performance and channel capacity in MIMO-wireless systems. Then, Section 4 presents the evaluation of the simulation results of multiple output differential unitary coding in MIMO-wireless systems. Finally, our main conclusions are summarized in Section 5.

\section{Multiple Output of DUSTF Coding in MIMO-Wireless Systems}

\subsection{MIMO-Wireless Channel Model}

The basic idea of Multiple input and multiple output (MIMO) systems is space time signal processing. The technique utilizes combination of signal processing in time domain, which is a natural dimension of digital communication data, and space domain, which is a utilization of array antenna distributed in space. The large spectral efficiencies associated with MIMO channels are based on multipath propagation with a rich scattering environment that provides independent transmission path between each transmit antenna and each receive antenna. On the other hand, MIMO takes advantage of random fading ([4], [5]) to increase transmission rates.

MIMO systems, employing several transmit and receive antenna at both ends, are able to provide more capacity than traditional single antenna systems. The capacity improvement based on the fact that the channels from a transmitter to a receiver follow independent path. If severe correlations present at the transmitter and/or the receiver side, for example, the capacity of the MIMO systems is degraded [6].

In order to make the availability of independent channel and to provide a simple multi-carrier transmission scheme, we suggest applying multiple output 
differential unitary space-time frequency (DUSTF) coding such as inner coding. Multiple output of DUSTF coding, however, are not designed to provide significant coding gain. Hence, powerful outer codes, i.e. combination of convolution code and block interleaving, can be concatenated with multiple output DUSTF coding to have a required coding gain.

We consider a MIMO-multiple output DUSTF wireless channel in which data are sent from $M_{T}$ transmit antennas to $N_{R}$ receive antennas. At transmitter, data are coded with $M_{T}$ parallel (multiple) output differential unitary encoder, one for each transmit antenna, which is processed into space frequency scheme. The resulting encoded symbols are mapped into a unit energy constellation $C_{c o n}$ and modulated onto a pulse wave with duration $T$ for sending over the channel.

The signal that arrives at each antennas of $N_{R}$ receive antennas is superposition of the $M_{T}$ fading transmitted signals and noise. We assume that the delay spread of the multipath is small and $K T$ is small compared with the channel coherence time, so that fading conditions can be considered constant over $K$ symbols. At each receive antenna, we utilize a demodulator which synchronously samples the output of a filter matched to the pulse waveform, producing $N_{R}$ decision statistics in each symbol interval. We assume that the elements in transmit and receive antennas are spaced so as to produce independent fading between each pair of transmit and receive antennas.

Under those conditions, MIMO-multilevel DUSTF wireless channel model in fading condition has signal equation in each receiver as follows:

$$
\begin{aligned}
& Z_{n_{R} k} \stackrel{\Delta}{=} \sum_{m_{T}=1}^{M_{T}} \sqrt{(S N R)_{M_{T}}} h_{n_{R} m_{T}} s_{m_{T} k}+w_{n_{R} k} \\
& n_{R}=1, \Lambda, N_{R} \quad m_{T}=1, \Lambda, M_{T} \quad k=1, \Lambda, K
\end{aligned}
$$

where:

$(S N R)_{M_{T}}=(S N R)_{n_{R}} / M_{T}$, where $(S N R)_{n_{R}}$ is the expected SNR at each receiver antenna,

$h_{n_{R} m_{T}}$ is the complex MIMO fading path gain from transmit antenna, $m_{T}$ to receive antenna, $n_{R}$,

$S_{m_{T} k}$ is the element of encoded transmit symbol matrix, $\mathbf{S}$,

$w_{n_{R} k}$ is a noise variable,

$M_{T} \quad$ is the number of antennas in the transmitter,

$N_{R} \quad$ is the number of antennas in the receiver, and 
$K \quad$ is the number of symbol durations used for a space-time code.

That channel equation above can be rewritten in an equivalent matrix-vector form as follows:

$\mathbf{Z}=\sqrt{(S N R)_{M_{T}}} \mathbf{H S}+\mathbf{W}$

where:

$\mathbf{Z}=\left\{z_{n_{R} k}\right\}$ is the $N_{R} \times K$ receive signal matrix,

$\mathbf{H}=\left\{h_{n_{R} m_{T}}\right\}$ is the $N_{R} \times M_{T}$ complex MIMO fading channel matrix,

$\mathbf{S}=\left\{S_{m_{T} k}\right\}$ is the $M_{T} \times K$ encoded transmit symbol matrix, and

$\mathbf{W}=\left\{w_{n_{R} k}\right\}$ is the $N_{R} \times K$ additive white Gaussian noise matrix.

The path gains $h_{n_{R} m_{T}}$ and noise variables $w_{n_{R} k}$ are therefore independent and identically distributed complex Gaussian random variables. Hence, probability density function (pdf) of any path gains is roughly equal, $p\left(h_{n_{R} m_{T}}\right)=p(h)$ as follows:

$$
p(h)=(1 / \pi) \exp \left(-|h|^{2}\right) \quad E\left\{h_{n_{R} m_{T}}\right\}=0 \text { for } n_{R} \neq m_{T}
$$

In addition, probability density function (pdf) of noise variables $w_{n_{R} k}$ :

$$
p_{w}(w)=\frac{1}{\pi \sigma_{w}^{2}} \exp \left(-w^{2} / \sigma_{w}^{2}\right) \quad \text { with } \sigma_{w}^{2}=E\left\{W^{2}\right\}
$$

\subsection{Multiple-output Differential Unitary Coding System Model}

Radio frequency in wireless communication systems is a limited natural resource, so that high data rate transmission or wide bandwidth of the information signal can be restricted by limited bandwidth of the radio signal. Consequently, it is necessary to implement transmission technique with high spectral efficiency [2]. In order to reduce mean processing time, it needs faster signal processing by applying parallel processing scheme. In addition, selective fading disturbs a wide bandwidth transmission signal. In high mobility conditions, it might be difficult to estimate the channel accurately. Channel estimation over fast fading environment requires more training symbols and complex computing power. Meanwhile, the perfect estimates of channel conditions assumption is acceptable if the channel changes slowly compared with the symbol rate, because the transmitters can send training symbols, which allow the receiver to estimate the channel accurately. 
We propose using a multicarrier differential inner coding scheme that is the multiple output of DUSTF encoding, to solve those problems. Furthermore, the implementation of multiple output of DUSTF coding scheme is a proposed solution for non-coherent channel transmission scheme, so that the wireless system could be applied regardless knowledge of the channel state. Figure 2 illustrates the multiple output of DUSTF group code in MIMO-wireless system.

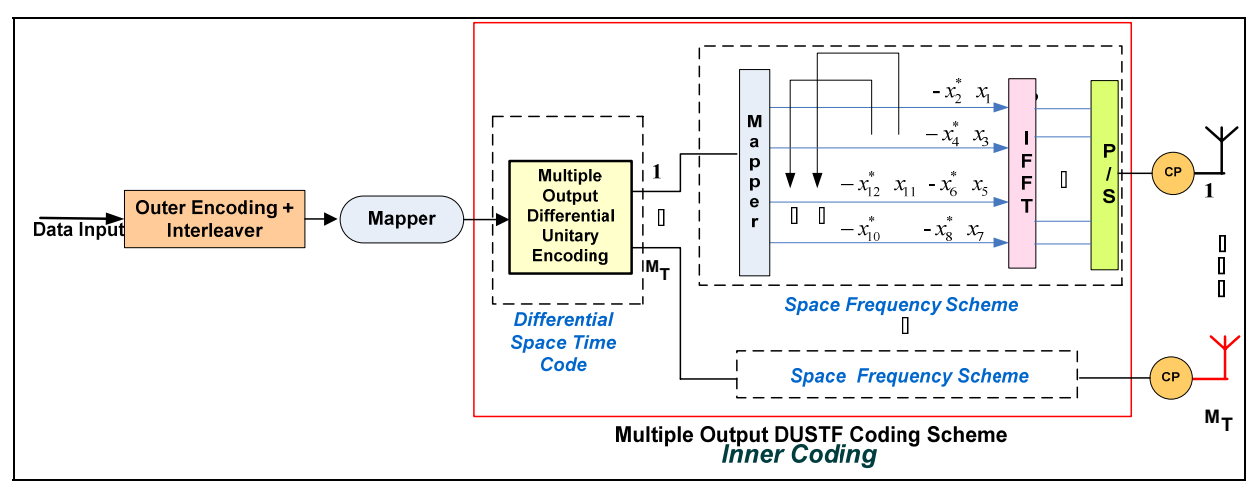

Figure 2 Multiple output of DUSTF group code in MIMO-wireless system.

We will describe the general idea of information signal processing at transmitter side from input data of MIMO wireless communication system with multiple output of DUSTF coding scheme. First, input data is processed at baseband stage, by means of channel coding as outer coding, i.e. convolutional code and interleaver, and then the data is fed to the mapper for signal constellation, i.e. $m$ BPSK. The next step is the multiple output of DUSTF coding part. The implementation of this inner coding scheme is unified with MIMO system, which can be applied with arbitrary $N_{R} \times M_{T}$ antennas system. The multiple output of DUSTF coding system consists of 2 main parts.

The first part of the inner coding employs a differential space-time encoding expanded from [3]. That is multiple output of differential unitary encoding subsystem. The parallel output of this subsystem is adjusted so that the parallel output data conforms to the number of antenna at transmitter. The application of parallel processing functions as spatial multiplexing will reduce the system performance theoretically, although it could give another advantage of system capacity. Nevertheless, in the proposed scheme, that poor performance is compensated by means of multiple output of differential unitary coding and multiple antennas, particularly at transmitter and receiver side. The differential unitary encoding guarantees the system performance, particularly at low SNR [7]. 
The second part is the multicarrier space frequency scheme to combat the selective fading problems, whereas the unitary coding technique is a solution to guarantee information signal quality. Moreover, multiple antennas and the space frequency scheme is capable of combating the problems of bandwidth restriction and selective coding condition. This scheme is employed to data symbol in each parallel data at every antenna arm of the MIMO wireless communication system. Then, the symbol data is entered into the IFFT system and ended with cyclic prefix insertion. The space frequency scheme in this new inner coding is similar to the modeling in [8], nevertheless there are several differences, i.e. in that paper differential transmit diversity from orthogonal designs using differential space time modulation scheme [2]. In this paper, however, this is the multiple output differential unitary encoding. Furthermore, the signal is transmitted simultaneously at each antenna of the MIMO wireless communication system. The rich environment condition will result in Rayleigh fading channel.

Receiver side will conduct the reverse process of that in transmitter. In each arm of receive antenna, cyclic prefix is removed, followed by FFT, soft output detector based on [8] to obtain symbols demapping of space frequency scheme. Moreover, signal equalization is applied at differential unitary decoding, by means of suboptimal maximum likelihood detector.

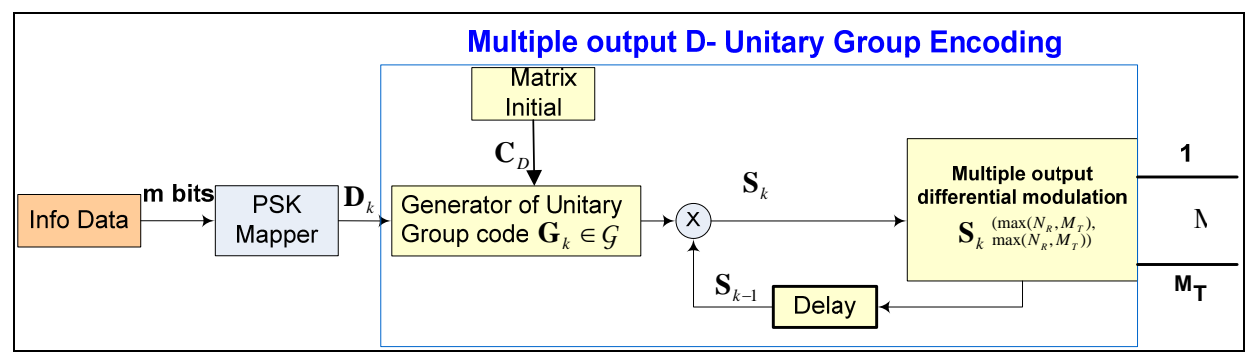

Figure 3 Multiple output of D-unitary group encoding subsystem.

Figure 3 explains the particular model of multiple output of differential unitary encoding subsystem. The $k^{\text {th }}$ symbol data, $\mathbf{D}_{k}$ from the mapper is processed at generator of the unitary group code stage, so that produce $\mathbf{G}_{k}$ which $\mathbf{G}_{k} \in \mathcal{G} \cdot \mathcal{G}$ is considered as the set of possible messages. The group structure code, $\mathcal{G}$ can be constructed for any number of transmit antennas and any constellation $\mathbf{C}_{c o n}$. In addition, $\mathcal{G}$ is any group of $n_{s} \times n_{s}$ unitary matrices, whose length of $n_{s}$ depends on the dimension of $N_{R} \times M_{T}$ antennas system. So that $\mathbf{G}^{H} \mathbf{G}=\mathbf{G G}^{H}=\mathbf{I}$ for all $\mathbf{G} \in \mathcal{G}$. For example: 
$2 \times 2$ antennas, BPSK mapper :

$4 \times 2$ antennas, QPSK mapper :

$$
\mathcal{G}=\left\{ \pm\left[\begin{array}{ll}
1 & 0 \\
0 & 1
\end{array}\right], \pm\left[\begin{array}{ll}
0 & 1 \\
1 & 0
\end{array}\right]\right\}
$$

$$
\mathcal{G}=\left\{ \pm\left[\begin{array}{llll}
1 & 0 & 0 & 0 \\
0 & 1 & 0 & 0 \\
0 & 0 & 1 & 0 \\
0 & 0 & 0 & 1
\end{array}\right], \pm\left[\begin{array}{cccc}
j & 0 & 0 & 0 \\
0 & j & 0 & 0 \\
0 & 0 & -j & 0 \\
0 & 0 & 0 & -j
\end{array}\right], \pm\left[\begin{array}{cccc}
0 & 0 & 0 & 1 \\
0 & 0 & 1 & 0 \\
0 & -1 & 0 & 0 \\
-1 & 0 & 0 & 0
\end{array}\right], \pm\left[\begin{array}{cccc}
0 & 0 & 0 & j \\
0 & 0 & j & 0 \\
0 & j & 0 & 0 \\
j & 0 & 0 & 0
\end{array}\right],\right\}
$$

\subsubsection{Particular model of Differential Encoding}

First, we assume that the system has a consideration: $M_{T x}=N_{R x}=n_{s}$ with encoding process as follows. An arbitrary $C_{u}$ is a unitary code, whose characteristic is:

$$
C_{u} C_{u}^{H}=n_{s} I \quad \text { for all } u=1, \Lambda, U
$$

where

()$^{H}$ is the conjugate transpose, and $n_{s}$ is number of symbols.

When perfect channel state information is available at the receiver, we can use the differential encoder, as below:

$$
\mathbf{S}_{k}=\mathbf{C}_{D} \mathbf{G}_{k} \quad k=1, \Lambda, K
$$

where $\mathbf{C}_{D}$ is an initial matrix coding. In that case, $\mathbf{C}_{D} \mathbf{G}_{k}$ is basically a space time block code with perfect channel estimation, which is available at the receiver. The collection of matrices of a group code of length $n_{s}$ over constellation $\mathbf{C}_{\text {con }}$ can be expressed as:

$$
\mathbf{C}_{D} \mathcal{G} \stackrel{\Delta}{=}\left\{\mathbf{C}_{D} \mathbf{G}_{k}: \mathbf{G}_{k} \in G\right\}
$$

Second, we assume that $\mathbf{C}_{D} \mathcal{G}$ is unitary group structure code, that is, if and only if $\mathbf{C}_{D} \mathbf{C}_{D}^{H}=n_{s} \mathbf{I} . \mathbf{C}_{D}$ can be chosen to be a $M_{T x} \times n_{s}$ matrix in $\mathbf{C}_{c o n}$. For example, for $m=n_{s}=2$ we can choose $\mathbf{C}_{D}$ to be any matrix that satisfies $\mathbf{C}_{D} \mathbf{C}_{D}^{H}=2 \mathbf{I}$. In this paper, $\mathbf{S}_{0}=\mathbf{C}_{D}$ is a $m \times n_{s}$ Hadamard matrix such that $\mathbf{C}_{D} \mathbf{G} \in \mathbf{C}_{\text {con }}^{m \times n_{s}}$ for all $\mathbf{G}_{k} \in G$, which be constrained with $|G|=2^{p}$. Therefore, the larger number of antennas (at transmitter or at receiver) is restricted to multiple powers of 2 (two). The fewer number of antennas (at transmitter or at receiver) 
may possibly have arbitrary number of antennas. If the cardinality of $\mathcal{G}$ is $|\boldsymbol{G}|$, then the rate of this code is:

$$
R=\frac{1}{n_{s}} \log _{2}|G| \operatorname{Bit} / \mathrm{s} / \mathrm{Hz}
$$

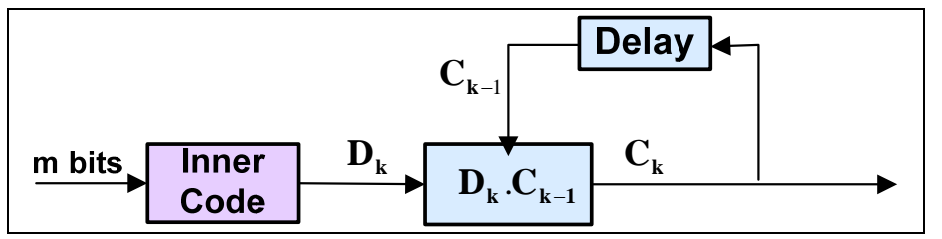

Figure 4 Differential modulation technique, based on block code or group code, in MIMO-wireless communication system.

The implementation of non-coherent channel transmission scheme with differential modulation is a good solution to overcome difficulties in estimating the response of the channel condition. In general, differential modulation of multiple antennas is based on group structure code, as illustrated in Figure 4. The technique is in a differential way similar to a traditional differential PSK [9]. For differential unitary coding, unitary space time signals are orthonormal in time across the antennas. It has been shown to be reliable in a Rayleigh fading channel where neither the transmitter nor the receiver knows the fading coefficients [3], [9], [10].

When no channel states information or no channel estimation scheme, with motivation from traditional differential modulation, PSK, we can use the differential encoder as follows (see Figure 3):

$$
\mathbf{S}_{k}=\mathbf{S}_{k-1} \mathbf{G}_{k} \quad k=1, \Lambda, K
$$

with initial code $\mathbf{S}_{0}=\mathbf{C}_{D}$.

The group structure code ensures that $\mathbf{S}_{k} \in \mathbf{C}_{D} \mathcal{G}$ whenever $\mathbf{S}_{k-1} \in \mathbf{C}_{D} \mathcal{G}$

\section{Symbol of \\ information \\ Generator of \\ Unitary Group Code}

Output of Differential modulation

$\begin{array}{cccc}\mathbf{D}_{1} & \mathbf{D}_{2} & \mathbf{D}_{3} & \Lambda \\ \mathbf{G}_{1} & \mathbf{G}_{2} & \mathbf{G}_{3} & \Lambda\end{array}$

$\mathbf{S}_{0}=\mathbf{C}_{D} \quad \mathbf{S}_{1}=\mathbf{S}_{0} \mathbf{G}_{1} \quad \mathbf{S}_{2}=\mathbf{S}_{1} \mathbf{G}_{2} \quad \mathbf{S}_{3}=\mathbf{S}_{2} \mathbf{G}_{3} \quad \Lambda$ 
The last step is the multiple output of differential modulation part. The parallel output of this subsystem is adjusted so that the parallel output data conforms to the number of antenna at transmitter.

\subsubsection{General model of Differential Encoding}

In general, the implementation of multiple output of DUSTF coding scheme in MIMO wireless system can have three specific conditions, which are:

- $M_{T x}=N_{R x}=n_{s}$, which has been explained as particular model of differential decoding

- $\quad M_{T x}<N_{R x}$ and $n_{s}=N_{R x}$, which has to generate initial code, $\mathbf{C}_{D}$ and unitary group code, $\mathcal{G}$ that is equal with encoding process in particular condition, $M_{T x}=N_{R x}=n_{s}$.

- $\quad M_{T x}>N_{R x}$ and $n_{s}=M_{T x}$, which $\mathbf{C}_{D}$ and $\mathbf{G}_{k}$ have to an $n_{s} \times n_{s}$ unitary matrices.

In $M_{T x}<N_{R x}$ and $n_{s}=N_{R x}$ conditions, we can choose initial code as follows: $\mathbf{S}_{k}\left(n_{s}, n_{s}\right)=\mathbf{S}_{k-1}\left(n_{s}, n_{s}\right) \cdot \mathbf{G}_{k}\left(n_{s}, n_{s}\right)$. However, we have $M_{T x}<N_{R x}$ antenna system, thus we have to select transmission code, $\widetilde{\mathbf{S}}_{k}\left(M_{T x}, n_{s}\right)$ from initial code, $\mathbf{S}_{k}\left(n_{s}, n_{s}\right)$. For example, possibility of transmission code for $3 \times 4$ antennas and BPSK mapper as follows:

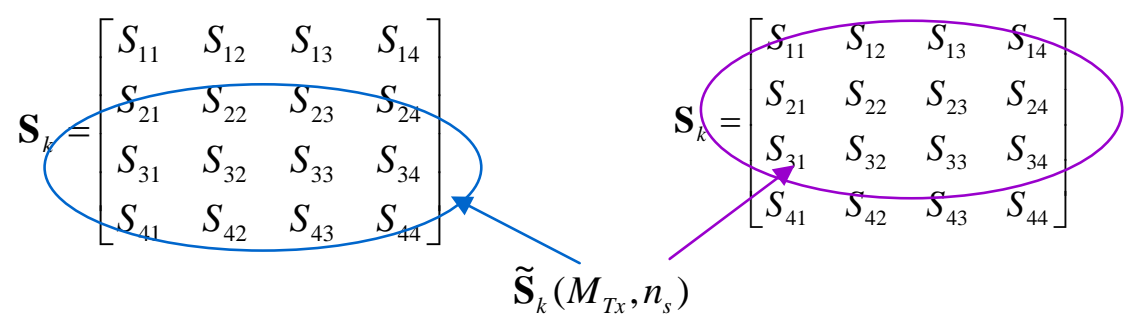

\subsubsection{General models of Differential Decoding}

In MIMO wireless communication system, the entire received sequence at a receiver for differential unitary group code is:

$$
\mathbf{Z}=\left[\mathbf{Z}_{0}: \Lambda: \mathbf{Z}_{K}\right]
$$


where $\quad \mathbf{Z}_{k} \stackrel{\Delta}{=} \sqrt{(S N R)_{M_{T x}}} \mathbf{H S}_{k}+\mathbf{W}_{k}$

$k=1, \Lambda, K$.

$(S N R)_{M_{T x}}$ is signal-to-noise ratio per receive antenna

$\mathbf{H}=\left\{h_{n_{R} m_{T}}\right\}$ is the $N_{R} \times M_{T}$ fading matrix.

Even for moderate values of $M_{T x}$ transmit antennas and $K$ block code, this receiver is quite complex. When the channel matrix, $\mathbf{H}$ is known at the receiver, the pdf of the received matrix given that $\mathbf{S}_{k}$ is transmitted is:

$$
p\left(\mathbf{Z}_{k} \mid \mathbf{H}, \mathbf{S}_{k}\right)=\frac{1}{\pi^{n_{S} N_{R x}}} \exp \left(-\operatorname{Tr}\left[\left(\mathbf{Z}_{k}-\sqrt{S N R_{M_{T x}}} \mathbf{H S}_{k}\right)\left(\mathbf{Z}_{k}-\sqrt{S N R_{M_{T x}}} \mathbf{H S}_{k}\right)^{H}\right]\right)
$$

The optimal receiver of a unitary code system is the maximum-likelihood (ML) detector, which reduces to the minimum Euclidean distance detector as:

$$
\begin{aligned}
\text { EuCl }_{d_{-} \min } & =\arg \max _{\text {Eucl }} p\left(\mathbf{Z} \mid \mathbf{H}, \mathbf{S}_{\text {Eucl }}\right) \\
& =\arg \min _{\text {Eucl }} \operatorname{Tr}\left[\left(\mathbf{Z}-\sqrt{S N R_{N x}} \mathbf{H S}_{\text {Eucl }}\right)\left(\mathbf{Z}-\sqrt{S N R_{N x}} \mathbf{H S}_{\text {Eucl }}\right)^{H}\right]
\end{aligned}
$$

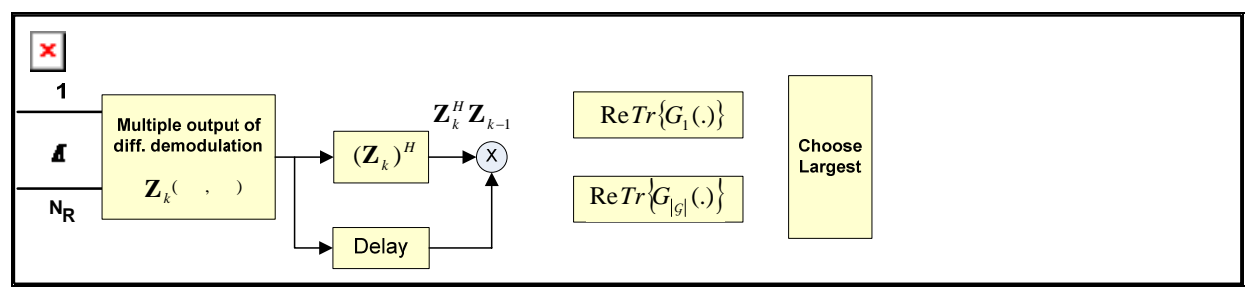

Figure 5 Multiple output of D-Unitary Space Time Frequency Decoding Scheme

In that differential unitary code system with no channel states information or no channel estimation case, it is natural to look for a receiver signal to estimate $\tilde{\mathbf{G}}_{k}$ using the last two received blocks (see Figure 5):

$$
\tilde{\mathbf{Z}}_{k} \stackrel{\Delta}{=}\left[\mathbf{Z}_{k-1}: \mathbf{Z}_{k}\right]
$$

On others word, to look for a receiver signal that estimates $\tilde{\mathbf{G}}_{k}$ from the received blocks, $\mathbf{Z}_{k}$ using the last two received blocks as follows:

$$
\tilde{\mathbf{G}}_{k}=\mathbf{Z}_{k}^{H} \mathbf{Z}_{k-1}
$$

First, we assume that the system has considered $M_{T x}=N_{R x}=n_{s}$ with encoding process as follows. We can generate initial received signal as: 


$$
\mathbf{Z}_{0}=\mathbf{C}_{D}\left(n_{s} n_{s}\right)
$$

Then, look for $\tilde{\mathbf{D}}_{1}$ from the entire received $\mathbf{Z}_{1}$ and the initial received signal $\mathbf{Z}_{0}$ :

$$
\tilde{\mathbf{D}}_{1}=\mathbf{G}_{1} \cdot \tilde{\mathbf{G}}_{1}=\mathbf{G}_{1} \cdot \mathbf{Z}_{1}^{H} \cdot \mathbf{Z}_{0}=\mathbf{G}_{1} \cdot \mathbf{Z}_{1}^{H} \cdot \mathbf{C}_{D}
$$

After that, search for $\tilde{\mathbf{G}}_{1}$ from the set of possible messages, that is $\mathcal{G}$ a unitary group code, followed by selecting $\tilde{\mathbf{D}}_{1}$ with $\mathrm{ML}$ detection:

$$
\tilde{\mathbf{D}}_{1}=\underset{\mathbf{G} \in \mathcal{G}}{\arg \max } \operatorname{Re} \operatorname{Trace}\left(\mathbf{G}_{1} \mathbf{Z}_{1}^{H} \mathbf{C}_{D}\right)
$$

In general, to look for $\tilde{\mathbf{D}}_{k}$ from the last two received blocks, $\mathbf{Z}_{k}$ and $\mathbf{Z}_{k-1}$ can be written by:

$$
\tilde{\mathbf{D}}_{k}=\mathbf{G}_{k} \cdot \tilde{\mathbf{G}}_{k}=\mathbf{G}_{k} \cdot \mathbf{Z}_{k}^{H} \cdot \mathbf{Z}_{k-1}
$$

Then, search for $\tilde{\mathbf{G}}_{k}$ from the set of possible messages, that is $G$ a unitary group code, followed by selecting $\widetilde{\mathbf{D}}_{k}$ with ML detection :

$$
\tilde{\mathbf{D}}_{k}=\underset{\mathbf{G} \in \mathcal{G}}{\arg \max } \operatorname{Re} \operatorname{Trace}\left(\mathbf{G}_{k} \mathbf{Z}_{k}^{H} \mathbf{Z}_{k-1}\right)
$$

Second, for the system consideration: $M_{T x}<N_{R x}$ and $n_{s}=N_{R x}$ to search $\tilde{\mathbf{G}}_{k}$ and to look for $\tilde{\mathbf{D}}_{k}$ from the entire received sequence, that is equal with decoding process in particular condition $M_{T x}=N_{R x}=n_{s}$

Third, for the system consideration: $M_{T x}>N_{R x}$ and $n_{s}=M_{T x}$, we can choose initial received signal as follows:

$$
\mathbf{Z}_{0}=\widetilde{\mathbf{C}}_{D}\left(N_{R x}, n_{s}\right)
$$

$\tilde{\mathbf{C}}_{D}\left(N_{R x}, n_{s}\right)$ can be selected from initial transmission code $\mathbf{C}_{D}\left(n_{s}, n_{s}\right)$, which use in the transmitter, e.g. $N_{R x} \times M_{T x}=3 \times 4$ antennas :

$\mathbf{C}_{D}\left(n_{s}, n_{s}\right)=\left[\begin{array}{cccc}1 & 1 & 1 & 1 \\ 1 & -1 & 1 & -1 \\ 1 & 1 & -1 & -1 \\ 1 & -1 & -1 & 1\end{array}\right]$

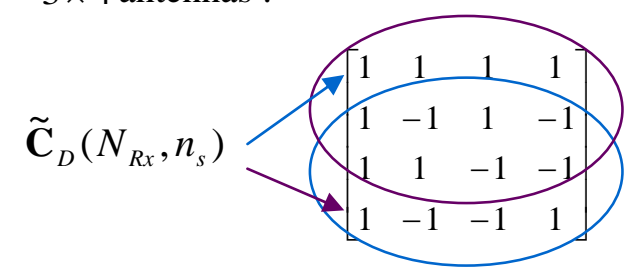


Then, to search $\tilde{\mathbf{G}}_{k}$ and to look for $\tilde{\mathbf{D}}_{k}$ from the entire received sequence, that is equal with decoding process in particular condition $M_{T x}=N_{R x}=n_{s}$.

\section{Channel Capacity}

Theoretically, the Shannon (ergodic) capacity of a single user time invariant channel is defined as the maximum mutual information between the channel input and output. The mutual information can be described as the reduction in the uncertainty of one random variable due to the knowledge of the other [11]. Meanwhile, the entropy of a random variable is a measure of uncertainty of the random variable. It is a measure of the amount of information required on the average to describe the random variable [12]. It will depend on the properties of the wireless channel used to express information from the transmitter to the receiver.

This maximum mutual information is shown by Shannon's capacity theorem that in random small error probability, the system can be transmitted over the channel with the maximum data rate.

$$
C_{\text {Shannon }}=B_{W} \log \left(1+\frac{P_{\text {average }}}{B_{W} N_{o}}\right)
$$

While the channel is time varying, channel capacity has multiple definitions. Depending on how the knowledge of the channel distribution or its state at the transmitter and/or receiver is. The capacity measurement can be conducted in several environments, i.e. minimum rate or average rate or maintained at a fixed constant over all channel distributions/states.

The MIMO channel capacity depends greatly on $\min \left(M_{T}, N_{R}\right)$, the statistical properties and correlations of the MIMO channel path gains. $M_{T}$ is number of antennas in transmitter and $N_{R}$ is number of antennas. At receiver, rich scattering environment can provide independent transmission paths from each transmit antenna to each receive antenna. On the other hand, strong correlation between the MIMO channel path gains can degrade the channel capacity significantly, which is the function of the scattering environment, the distance between transmitter and receiver, the antenna configurations, and the Doppler spreads [11].

When perfect channel knowledge at the receiver by assuming maximum ratio combining at the receiver and the transmitter has no knowledge of the channel, it is optimal to consistently distribute the available power $P_{T}$ between the 
transmit antennas [13], i.e. $\mathbf{P}_{\text {signal }}=\frac{P_{T}}{M_{T}} \mathbf{I}_{n_{s}}$. We assume that the noise is uncorrelated among branches of MIMO, so the noise covariance matrix at receiver $\mathbf{P}_{\text {noise }}=\sigma_{n}^{2} \mathbf{I}_{n_{R}}$. Therefore, the MIMO fading channel capacity can be written as [14]:

$$
\begin{aligned}
C_{\text {MIMO }} & =h_{d e}(\mathbf{Z})-h_{d e}(\mathbf{W}) \\
& =\log _{2}\left[\operatorname{det}\left(\mathbf{H P}^{S} \mathbf{H}^{H} \mathbf{P}^{n}\right)\right]-\log _{2}\left[\operatorname{det} \mathbf{P}^{n}\right] \\
& =\log _{2}\left[\operatorname{det}\left(\mathbf{I}_{N_{R}}+\frac{P_{T}}{\sigma_{n}^{2} M_{T}} \mathbf{H H}^{H}\right)\right]
\end{aligned}
$$

where:

$P_{T} \quad$ is the available power,

$\mathbf{P}^{S} \quad$ is the distributed power of the available power among the transmit antennas,

$\mathbf{P}^{n} \quad$ is the noise covariance matrix with assuming that the noise is uncorrelated between branches,

$h_{d e}($.$) is denoting differential entropy (entropy of a continuous random$ variable), and

H is MIMO channel matrix, $M_{T} \times N_{R}$

with an eigenvalue decomposition of the matrix product $\mathbf{H} \mathbf{H}^{H}=\mathbf{E} \Lambda \mathbf{E}^{H}$, where $\mathbf{E}$ is the eigenvector matrix with orthonormal columns with $M_{T} \times N_{R}$ dimension and $\Lambda$ is a diagonal matrix with with $N_{R} \times M_{T}$ dimension and the eigenvalues on the main diagonal $\left(N_{R} \times N_{R}\right)$. The MIMO system capacity may be written as follows:

$$
C_{\text {MIMO }}=\sum_{l=1}^{k} \log _{2}\left(1+\frac{P_{T}}{\sigma_{n}^{2} M_{T}} \lambda_{l}\right)
$$

where :

$k=\operatorname{rank}(\mathbf{H}) \leq \min \left(M_{T}, N_{R}\right)$,

$\sum$ is a real matrix, and

$\operatorname{det}\left(\mathbf{I}_{A B}+\mathbf{A B}\right)=\operatorname{det}\left(\mathbf{I}_{B A}+\mathbf{B A}\right)$. 
$\lambda_{l}$ are the non zero eigenvalue of matrix product $\mathbf{H H}^{H}$. In the orthogonal channel path case, $\lambda_{l}=$ (diversity_order) $E\left\{h_{m_{T}} h_{m_{T}}^{\cdot}\right\}=N_{R} E\left\{h_{m_{T}} h_{m_{T}}^{\cdot}\right\} \quad$ with $m_{T}=1,2, \Lambda, M_{T}$. In the real world case, correlated channel path gains occurr so the single non zero eigenvalue of response $\mathbf{H}$ is $\lambda_{l}=N_{R} E\left\{h_{m_{T}} h_{m_{T}}^{\cdot}\right\}=N_{R} M_{T}$. If we assume that $\mathbf{H}$ is a random process, we can identify the channel at the receiver by using a training sequence, assuming that the training sequence does not cost any capacity.

In practice, the realization of high MIMO capacity is responsive not only to the fading correlation between individual antennas but also to the rank performance of the channel. High rank behavior has been heavily linked to the existence of a rich scattering environment and a little correlation between the channel paths, for example in Rayleigh fading model. The MIMO channels have a diversity gain defined by the rank of $\mathbf{H H}^{*}$. The maximum achievable diversity gain is $\operatorname{rank}\left(H H^{*}\right)=\min \left(M_{T}, N_{R}\right)$. The maximum diversity gain and the upper limit for the capacity of MIMO channels is represented by the orthogonal channel gain case. Assuming $M_{T}$ columns of $\mathbf{H}$ are orthogonal and the entries of $\mathbf{H}$ are normalized to power unit, the eigenvalues of $\mathbf{H H}^{H}$ are $\lambda_{l}=N_{R}$ for $l=1,2, \Lambda, M_{T}$. The capacity of the high-rank MIMO channel can be rewritten from equation (25) and (26) as:

$$
\begin{aligned}
& C_{\text {high_rank }}=\sum_{l=1}^{k=\min \left(M_{T}, N_{R}\right)} \log _{2}\left(1+\frac{P_{T}}{\sigma_{n}^{2} M_{T}} \lambda_{l}\right)
\end{aligned}
$$

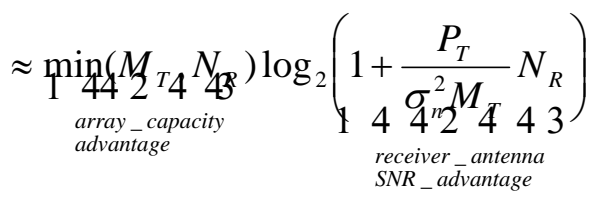

On the other hand, Low rank performance has been closely linked to the fewer scattering environment and a strong correlation between each channel path, for example in Ricean fading model with large $\mathrm{K}$ factor. The correlation characteristics determine the rank of $\mathbf{H} \mathbf{H}^{H}$, which eventually determines the diversity advantage. A fully correlated $\mathbf{H}$ matrix provides no diversity gain over the single antenna case and gives the all one's matrix with dimension $M_{T} x N_{R}$. In high correlated channel condition, all gains $h_{i j}$ are approximately equal, and $\mathbf{H}$, a multiple of the all-one matrix, has a single non zero singular value 


$$
\lambda_{\text {low_rank }}=N_{R} \sum_{l=1}^{M_{T}} E\left(h_{l l}^{H} h_{l l}\right) \approx M_{T} N_{R}
$$

The capacity of the low-rank MIMO channel can be expressed as:

$$
\begin{aligned}
C_{\text {low_rank }} & =\sum_{l=1}^{k=\min \left(M_{T}, N_{R}\right)} \log _{2}\left(1+\frac{P_{T}}{\sigma_{n}^{2} M_{T}} \lambda_{l}\right) \\
& \approx \log _{2}\left(1+\frac{P_{T}}{\sigma_{n}^{2}} N_{R}\right)
\end{aligned}
$$

In Low-SNR MIMO channel, $\left(P_{T} / \sigma_{n}^{2}\right)$, is low; with a Taylor series approximation $\log (1+x) \approx x$ for small values of $x$. Both of the capacity of the high-rank MIMO channel for small SNR, $C_{h i g h \_S N R}$, and the capacity of the low-rank MIMO channel for small SNR, $C_{\text {low_SNR }}$, shall be expressed as:

$$
\begin{aligned}
& C_{\text {high_SNR }} \approx \min \left(N_{R}, M_{T}\right) \frac{P_{T}}{\sigma_{n}^{2} M_{T}} N_{R} \\
& C_{\text {low_SNR }} \approx \frac{P_{T}}{\sigma_{n}^{2}} N_{R}
\end{aligned}
$$

When no channel information is required by receiver, the MIMO channel capacity takes expectation over an instantaneous channel, as follows:

$$
C_{\text {average }}=E\left\{\log _{2} \operatorname{det}\left(I_{N_{R}}+\frac{P_{T}}{\sigma_{n}^{2} M_{T}} \mathbf{H H}^{H}\right)\right\}
$$

A framework for differential modulation, which is multilevel DUSTF coding, is no channel knowledge at the receiver. Then, the capacity of a MIMO wireless fading channel using multilevel DUSTF coding can be written as:

$$
C=\frac{K}{T} \cdot \log _{2}\left(1+\frac{P_{T}}{\sigma_{n}^{2} M_{T}}\|\mathbf{H}\|_{F}^{2}\right)
$$

where

$K$ is code number of block code used for a space time code, unitary code,

$T \quad$ is number of the time slots in block code, and

$\|\mathbf{H}\|_{F}^{2}$ is the squared Frobenius norm of matrix $\mathbf{H}$. 


\section{$4 \quad$ Discussion of the Simulation Results}

In this paper, the study is focused on the downlink transmission, i.e. signal transmission from base station to mobile user. Our simulation is emphasized in a research to explore the response of MIMO wireless communication system to the proposed inner coding scheme. The objectives are to answer high data rate problems, selective fading problems, and the difficulty to estimate response of the channel in high-speed mobility.

By considering those problems, in this section we present simulation results supporting those considerations. Simulation is focused on the utilization of proposed inner coding scheme in MIMO wireless communication systems in non-coherent channel transmission scheme. Accordingly, as comparison, we also present coherent channel transmission scheme with perfect channel acknowledgement. In all simulation scenarios, we use frequency of $5 \mathrm{GHz}$, convolutional code rate $1 / 2$, Viterbi decoder at receiver and 256 bits per data block. Modulation system could be any scheme, MPSK or MQAM, but in our simulation, BPSK is implemented, with power efficiency consideration. It is assumed that the simulation results of all multiple-output DUSTF coding schemes are observed in fading channel condition by applying suboptimal ML detection at receiver.

Firstly, the influence of the proposed inner coding scheme application to the MIMO wireless system with non-coherent channel transmission scheme in fading channel condition is shown for various number of subcarrier in both nonmobility and high mobility of users. As comparison, the BER system performance in coherent channel transmission scheme with perfect channel acknowledgement and high mobility of users is also presented. It is assumed that the simulation results of all multiple-output DUSTF coding schemes are observed in the same transmission parameters and fading channel condition.

Figure 6 shows that the optimal number of sub-carrier in each fading channel condition is different. In these simulation cases, the 128 subcarrier is the optimal number for non-mobility of users. However, the 64 sub-carriers is the optimal number for high mobility of users. In the simulation cases of high mobility condition, at low SNR the BER performance of MIMO wireless system performance with coherent channel transmission scheme (assuming in the perfect estimation condition) has slightly better performance than the system with non-coherent channel transmission scheme. However, at medium or high SNR, the BER performance of MIMO wireless system both with the perfect estimation condition and with non-coherent channel transmission scheme, is very similar. 

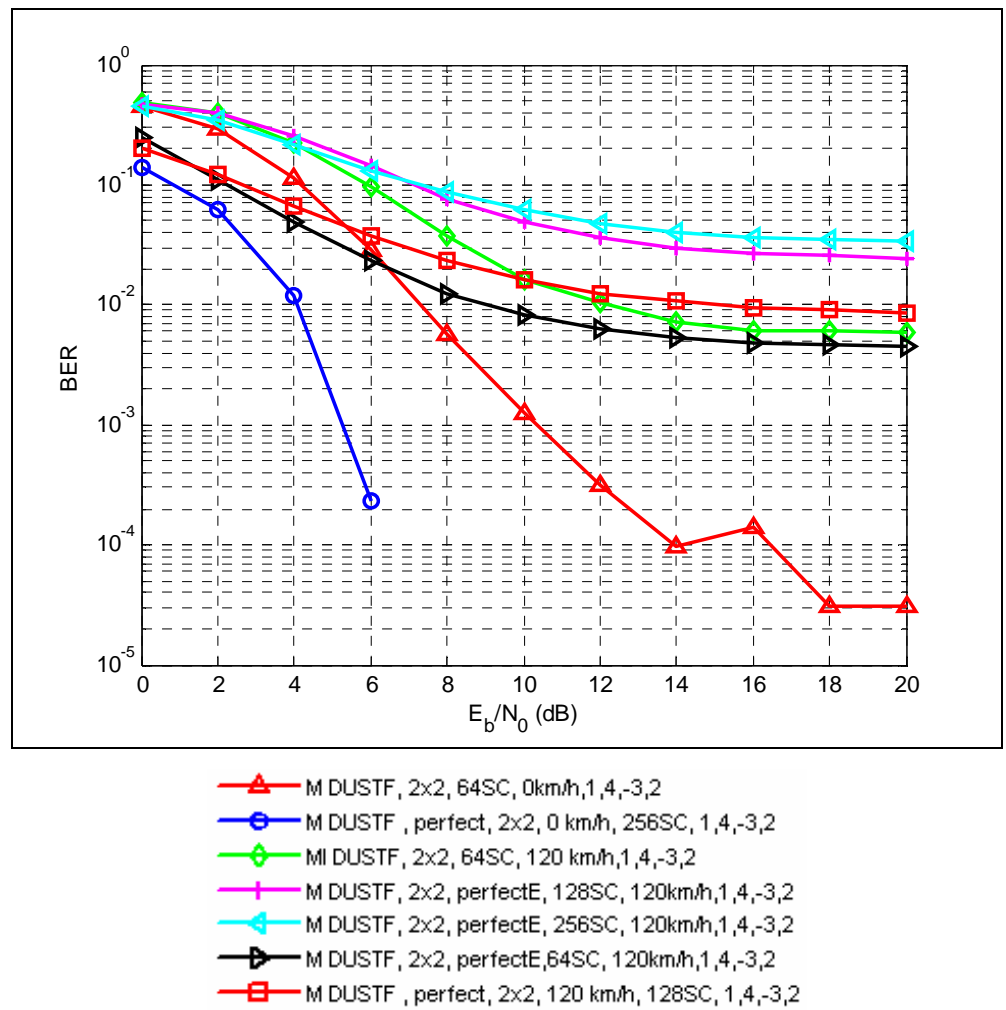

Figure 6 The BER performance of multiple output DUSTF coding scheme in MIMO wireless system for various number of sub-carrier in: both non and high mobility of users.

Secondly, we explore that the multilevel DUSTF coding with the 64 subcarriers and non-coherent channel transmission scheme in the selective fading channel condition. Then, we compare the proposed inner coding with other inner coding schemes for both non-mobility and high mobility of users. In these cases, the other inner coding schemes are STBC, OFDM, and D-STF, which had been observed in [2]. It is assumed that the simulation results of all inner coding schemes are observed in the same transmission parameters, and the same fading channel condition. 


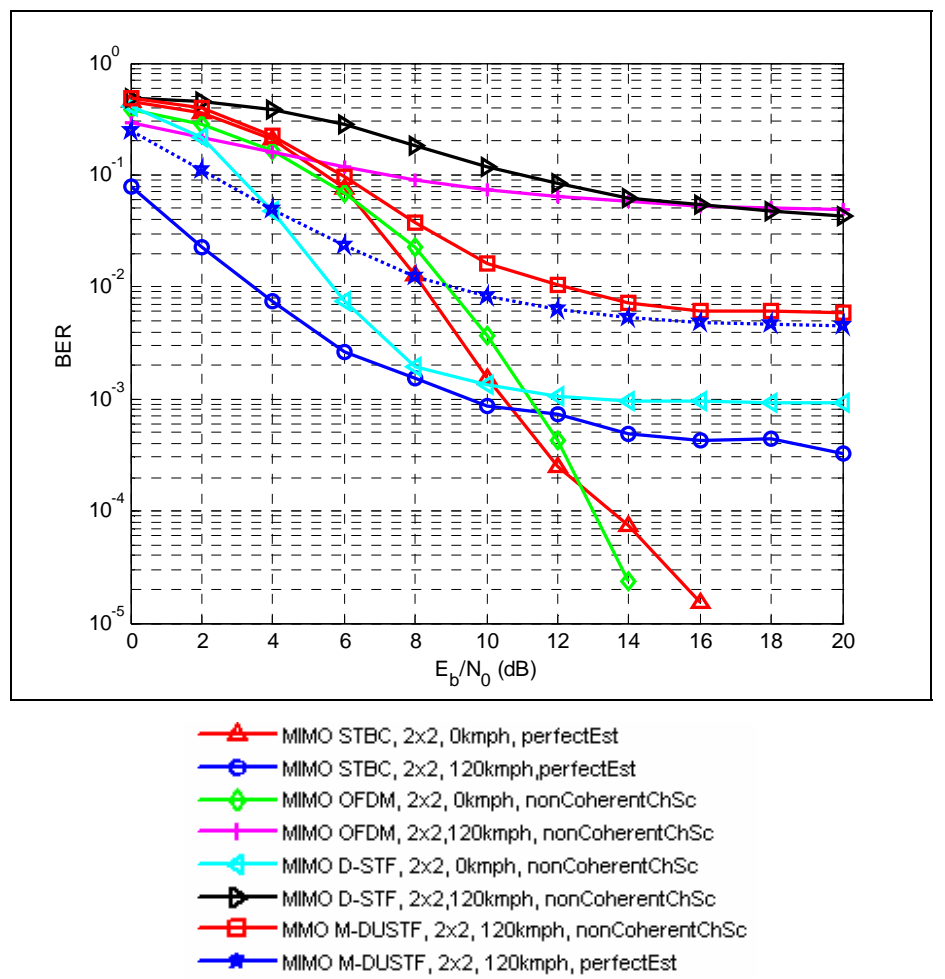

Figure 7 The BER performance comparisons of multiple output DUSTF coding with non coherent channel transmission and other inner coding schemes in MIMO wireless system.

Figure 7 shows the comparison among the MIMO wireless systems performance, i.e. MIMO-STBC system with perfect channel estimation; MIMO-OFDM system, MIMO-D STF system and MIMO-multiple output DUSTF coding system with non-coherent channel transmission scheme. This figure shows that in similar channel condition with non-coherent channel transmission scheme, i.e. fading channel and in high mobility conditions, then the MIMO-multiple output DUSTF coding wireless systems has better performance than the system that uses other modulations. Furthermore, at high SNR and high mobility of users, applying coherent channel transmission with perfect estimation acknowledgement does not improve the system performance significantly. The system performance both for coherent and non-coherent channel transmission schemes is very similar.

Thirdly, we consider the effect of receive antenna diversity or transmit antenna diversity to the performance of the proposed inner coding scheme. This coding scheme is studied in the MIMO wireless system with non-coherent channel 
transmission scheme, i.e. observing the influence of 2 transmit antenna and $n$ receive antennas, and also $m$ transmit antennas and 2 receive antennas. It is assumed that the simulation results of the proposed inner coding schemes are observed under the same transmission parameters and fading channel condition.

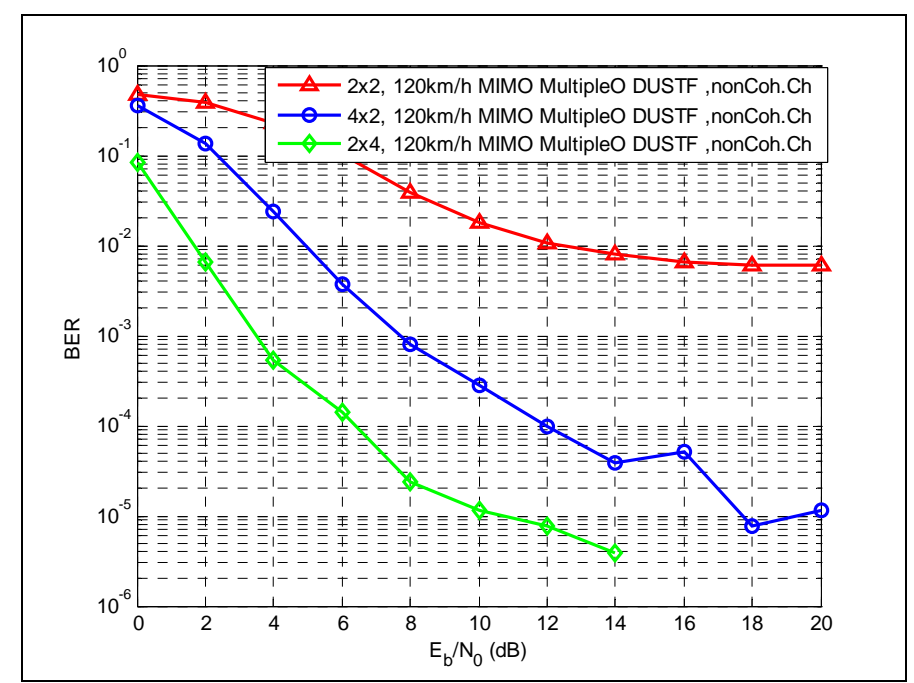

Figure 8 The effect of $n$ receive antennas diversity or $m$ transmit antennas diversity to the performance of MIMO-multiple output DUSTF wireless system.

Figure 8 shows that generally increasing the number of receive antennas provide better performance than increasing the number of transmit antennas, although it gives another advantage, i.e. system capacity improvement. Applying greater number of transmit or receive antennas at BTS could be a good solution for compensation of the performance deterioration in high mobility conditions.

Finally, we investigate the channel capacity of multiple output DUSTF coding scheme in the MIMO wireless system with coherent channel transmission scheme, which is done in Low Rank and High Rank environment for high mobility of users. As comparison, the channel capacity of the other schemes is also presented, i.e. MIMO and MIMO-STBC, which had been observed in [14].

Figure 9 shows that the MIMO wireless system with spatial multiplexing scheme has higher channel system capacity than the system with an inner coding, i.e. STBC and multiple output DUSTF coding for both in Low Rank and High Rank environment. In these cases, MIMO-STBC is applied with perfect estimation acknowledgement by the receiver side. Meanwhile, MIMOmultiple output DUSTF coding is applied with non-coherent channel 
transmission scheme. However, the wireless system capacity is very similar for both the MIMO-STBC and the MIMO-multiple output DUSTF coding system.

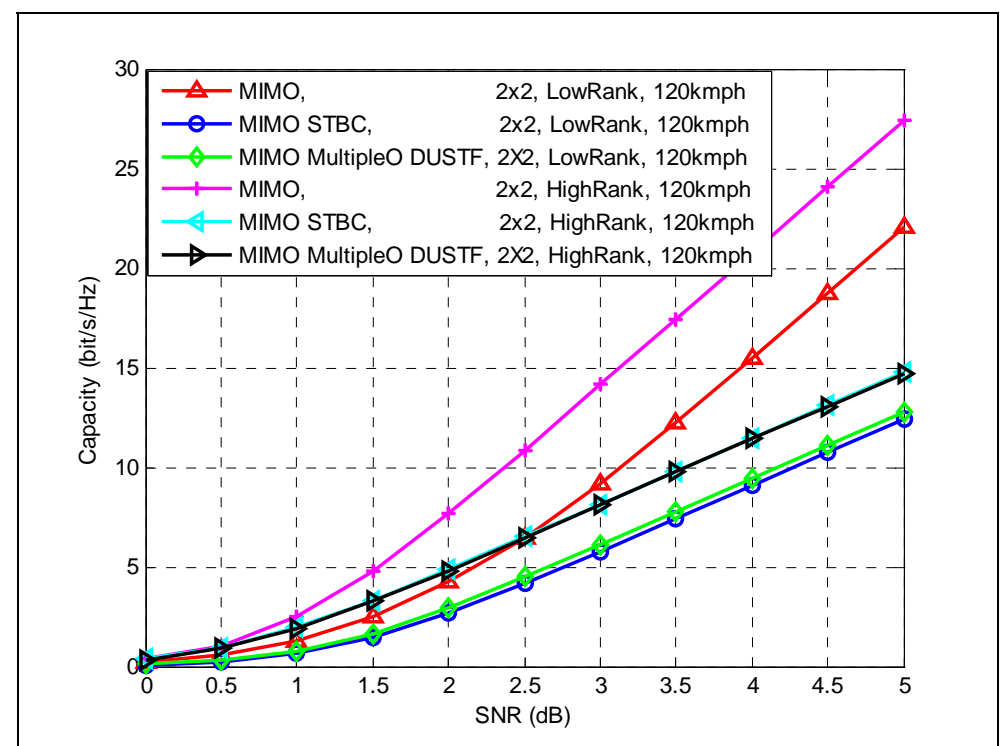

Figure 9 The channel system capacity comparisons between the multiple output DUSTF coding and the other schemes for high mobility of users in MIMO wireless system.

\section{$5 \quad$ Conclusion}

From the study results, it is concluded that the proposed inner coding scheme with non-coherent channel transmission scheme will provide a good system performance. Especially at high SNR for high mobility of users with noncoherent channel transmission scheme in selective fading channel condition, the system shows better performance than the other inner coding schemes. Applying greater number of transmit or receive antennas and optimal number of sub-carrier at BTS could compensate performance deterioration in high mobility conditions. The MIMO-multiple output DUSTF coding wireless system also has a good channel capacity.

Nevertheless, the proposed scheme in MIMO wireless system has limitations, which do not accommodate various requirements: bandwidth usage, QoS or channel response for every user. There are also trade-offs among the system performances, channel capacity and system complexity. Accordingly, that consideration can be a research subject in the future. 


\section{References}

[1] Patzold, M., Mobile fading Channels, John Wiley \& Sons, England, 2002.

[2] Astuti, R.P., Suksmono, A.B., Sugihartono, Kurniawan, A., The Performance of Non-Coherent Space Time Coding in MIMO-wireless Systems, Proc. of TSSA 2006 \& WSSA 2006, International Joint Conf, 278-284, Des. 2006.

[3] Hughes, B.L., Differential Space Time Modulation, IEEE Transactions on Information Theory, 46, no.7, 2567-2578, November 2000.

[4] Foschini, G.J., Layered Space Time Architecture for Wireless Comm. in a Fading Environment When Using Multi Element Antennas, Bell Labs Technical Journal, 41-59, autumn 1996.

[5] Foschini, G.J. \& Gans, M.J., On Limits of Wireless Communications in a Fading Environment when using Multiple Antennas, Wireless Personal Communications, 6, 311-335, 1998.

[6] Gesbert, D., Shafi, M., Shiu, D.S., Smith, P. \& Naguib, A., From Theory to Practice: An Overview of MIMO Space Time Coded Wireless Systems, IEEE Journal on Selected Areas in Comm., I, 21, 281-302, April 2003.

[7] Li, H., Differential ST Modulation over Frequency Selective Channels, IEEE Transactions on Signal Processing, 53, no. 6, 2228-2242, June 2005.

[8] Bauch, G., Differential Space Time Frequency Transmit Diversity in OFDM, Proc. Of International Symposium on Wireless Personal Multimedia Communications, October 2003.

[9] Hochwald, B.M. \& Sweldens, W., Differential Unitary Space Time Modulations, unpublished, February 2000.

[10] Hochwald, B.M \& Marzetta, T.L., Unitary Space-Time Modulation for Multiple-Antenna Communications in Rayleigh Flat Fading, IEEE Transactions on Information Theory, 46, no.2, 543-546, March 2000.

[11] Goldsmith, A., Jafar, A.A, Jindal, N. \& Vishwanath, S., Capacity limits of MIMO channels, IEEE Journal on Selected Areas in Comm., II, 21, 684-700, April 2003.

[12] Holter, B., Capacity of multiple-input multiple output (MIMO) systems in wireless communications, NTNU, 2002.

[13] Vucetic, B. \& Yuan, J., Space-Time Coding, John Willey \& Sons Ltd., West Succex, England, 2003.

[14] Astuti, R. P., Tati, L.R.M., Sugihartono, \& Suksmono, A.B., High and Low Rank MIMO Ch. Capacity on MIMO-Wireless Comm. Systems, Proc. of International Conference on Applied Mathematics (ICAM05), August 2005. 